\title{
Generating coherent states of entangled spins
}

\author{
Hongyi Yu, Yu Luo, and Wang Yao* \\ Department of Physics and Center of Theoretical and Computational Physics, The University of Hong Kong, Hong Kong, China
}

(Received 21 April 2011; published 26 September 2011)

\begin{abstract}
A coherent state of many spins contains quantum entanglement, which increases with a decrease in the collective spin value. We present a scheme to engineer this class of pure state based on incoherent spin pumping with a few collective raising or lowering operators. In a pumping scenario aimed for maximum entanglement, the steady state of $N$-pumped spin qubits realizes the ideal resource for the $1 \rightarrow \frac{N}{2}$ quantum telecloning. We show how the scheme can be implemented in a realistic system of atomic spin qubits in an optical lattice. Error analysis shows that high-fidelity state engineering is possible for $N \sim O(100)$ spins in the presence of decoherence. The scheme can also prepare a resource state for the secret sharing protocol and for the construction of the large-scale Affleck-Kennedy-Lieb-Tasaki state.
\end{abstract}

DOI: 10.1103/PhysRevA.84.032337 PACS number(s): 03.67.Bg, 37.10.Jk, 42.50.Dv, 76.70.Fz

\section{INTRODUCTION}

A coherent state in quantum mechanics usually refers to a specific type of quantum state with minimum uncertainty. It was first discovered in the context of an oscillator field and has found wide applications in quantum optics [1-4]. A quantum harmonic oscillator in a coherent state most closely resembles the behavior of a classical oscillator. The notion was later generalized to spin systems [5-8]. In an ensemble of $N$ spin- $I$ particles, the term spin coherent state (or atomic coherent state) is used to denote the states where the collective spin $\hat{\mathbf{J}} \equiv \sum_{n} \hat{\mathbf{I}}_{n}$ has the minimum uncertainty [8]. Such states can easily be identified in the basis $|J, \mu, \vec{\lambda}\rangle$, which are eigenstates of $\hat{J}^{2}$ and $\hat{J}_{z}$ with eigenvalues $J(J+1)$ and $\mu$, respectively, and $\vec{\lambda}$ denotes additional quantum numbers to provide a complete set of labels. The collective spin $J$ gets every value from $N I$ down to 0 (or $\frac{1}{2}$ ) in integer steps, and for each collective spin value $J$, the magnetic quantum number $\mu=$ $-J,-J+1, \ldots, J$. One can easily show that the minimum uncertainty relation $\left\langle\left(\Delta \hat{J}_{x}\right)^{2}\right\rangle\left\langle\left(\Delta \hat{J}_{y}\right)^{2}\right\rangle=\frac{1}{4}\left\langle\hat{J}_{z}\right\rangle^{2}$ is satisfied for all extremal states $|J, \mu=-J, \vec{\lambda}\rangle$ in this basis. Hence, these states and their rotations generated by $\hat{\mathbf{J}}$ are the spin coherent states (SCSs) [8]. Interestingly, two contrary characters coexist in these states: the most classical collective spin behavior and the fundamentally nonclassical phenomenon of quantum entanglement. For every $J<N I$, there is a degenerate set of $|J,-J, \vec{\lambda}\rangle$ with identical collective properties and distinct entanglements where the number of unentangled spins is upper bounded by $\frac{J}{I}[9]$.

The preparation of SCSs has been possible only in limited cases. The $J=N I$ SCS, nondegenerate and unentangled, is obtained when all spins are fully polarized. Most experimental studies of spin squeezing start in this state. Schemes were also proposed to populate a mixed state of singlets ( $J=0$ SCS) by collective pumping [10] and to select out a singlet by projective measurement in a scattering model [11]. Engineering a purestate SCS of an arbitrary $J$ value is a challenge but of multifold significance. It is the sufficient condition for initialization into a decoherence-free subsystem for robust quantum computation

*wangyao@hkucc.hku.hk under strong collective decoherence [12]. SCSs of $J \ll N I$ are resources of large-scale entanglement with potential uses in one-way quantum computation $[13,14]$. The ability to access an SCS of entangled spins also opens up a new realm for the study of the interrelation between collective spin behaviors and quantum correlations in a spin ensemble $[15,16]$.

In this paper, we propose control schemes for engineering pure-state SCSs of an arbitrarily specified collective spin value in a general spin ensemble. The schemes are based on incoherent spin pumping of the $N$ target spins and a set of ancilla spins by a few (e.g., three) collective raising and lowering operators. The desired pure state is obtained with an $N$-independent probability by a single projective measurement on the steady state of the pumping, and the success rate approaches $100 \%$ with $O(10)$ cycles of pump plus measure. In a simplified pumping scenario aimed for maximum entanglement, the steady state of $N$-pumped spin qubits (without measurement projection) realizes the ideal resource for $1 \rightarrow \frac{N}{2}$ optimal quantum telecloning [17]. We show how the scheme can be implemented in the realistic system of atomic spin qubits trapped in an optical lattice, where the collective spin pumping is realized by Stokes or anti-Stokes light scattering. Error analyses show that high-fidelity state engineering is possible for up to $N \sim O(100)$ atomic spin qubits in the presence of control errors and decoherence. This is a concrete example of using simple and robust irreversible dynamics to prepare a desired complicated quantum state [18-23]. The scheme can also prepare a resource state for the secret sharing protocol [24] and for the efficient construction of a large-scale Affleck-Kennedy-Leib-Tasaki (AKLT) state with applications in one-way quantum computation [25-27].

\section{GENERAL SCHEME}

The key to the state engineering approach by irreversible dynamics is to design the dissipative controls under which the system saturates with the desired state vectors. Here, we utilize the spin-pumping process that drives a spin system toward a mixture of all singlets connected to the initial state by the pumping operators [10]. If a target spin ensemble is in a singlet with a spin- $J$ ancilla, its collective spin value also must equal $J$. With a proper constraint from conserved 
quantum numbers, the singlet can be unique from which the desired pure-state SCSs of the target spins can be obtained.

The target spins are divided into two subgroups with collective spins $\hat{\mathbf{j}}_{A}$ and $\hat{\mathbf{j}}_{B}$, respectively, and the collective spin of $2 J$ spin- $\frac{1}{2}$ ancillas is denoted by $\hat{\mathbf{j}}_{\beta}$. When an inhomogeneous collective operator of the form $\hat{J}_{i}^{+}=c_{A} \hat{j}_{A}^{+}+c_{B} \hat{j}_{B}^{+}+c_{\beta} \hat{j}_{\beta}^{+}$ acts on an SCS, the final state can generally be written as

$$
\hat{J}_{i}^{+}\left|J_{T},-J_{T}, \vec{\lambda}\right\rangle=\sum_{\Delta, \vec{\lambda}^{\prime}} \chi_{J_{T}, \vec{\lambda}}^{J_{T}+\Delta, \vec{\lambda}^{\prime}}\left|J_{T}+\Delta,-J_{T}+1, \vec{\lambda}^{\prime}\right\rangle,
$$

where the first two quantum numbers in the kets denote the total spin and the $z$ component of $\hat{\mathbf{J}}_{T}=\hat{\mathbf{j}}_{A}+\hat{\mathbf{j}}_{B}+\hat{\mathbf{j}}_{\beta}$, respectively. The calculation of coefficients $\chi$ is straightforward by expanding the collective spin states in terms of the common eigenstates of $\hat{j}_{A}^{2}, \hat{j}_{A}^{z}, \hat{j}_{B}^{2}, \hat{j}_{B}^{z}, \hat{j}_{\beta}^{2}$, and $\hat{j}_{\beta}^{z}$. We find that only the $\Delta=0, \pm 1$ transitions are allowed [10], and the ratio between transition rates $\Delta= \pm 1$ is

$$
\left|\chi_{J_{T}+1, \vec{\lambda}}^{J_{T}, \vec{\lambda}^{\prime}}\right|^{2}=\left(J_{T}+1\right)\left(2 J_{T}+1\right)\left|\chi_{J_{T}, \vec{\lambda}^{\prime}}^{J_{T}+1, \vec{\lambda}}\right|^{2}
$$

Consider the incoherent strong pump by $\hat{J}_{T}^{-}$, which results in a mixture of SCSs of $\hat{\mathbf{J}}_{T}$ and the weak pump by the inhomogeneous operator $\hat{J}_{i}^{+}$, which then causes transitions between these SCSs with the effective rate $\propto|\chi|^{2}$ and the selection rule $\Delta=0, \pm 1$. From Eq. (2), we can see that the $\Delta=-1$ transition is much faster than the $\Delta=1$ one between any such pairs of states. Thus, the pump will saturate the target and the ancilla spins to singlets where $J_{T}$ is minimized. With the target and ancilla spins initialized on the fully polarized state, the quantum numbers $j_{A}=N_{A} I, j_{B}=N_{B} I$, and $j_{\beta}=J$ are all conserved by the pump operators. Only one singlet exists under this constraint,

$$
\left|S_{A B \beta}\right\rangle \equiv \sum_{\mu=-J}^{J}(-1)^{J-\mu}\left|J, \mu, j_{A}, j_{B}\right\rangle_{A B} \otimes|J,-\mu\rangle_{\beta},
$$

where $\left|J, \mu, j_{A}, j_{B}\right\rangle_{A B}$ denotes eigenstates of $\left(\hat{\mathbf{j}}_{A}+\hat{\mathbf{j}}_{B}\right)^{2}$ and $\hat{j}_{A}^{z}+\hat{j}_{B}^{z}$ with eigenvalues $J(J+1)$ and $\mu$, respectively.

Figure 1(a) presents a simulation of the spin pump using the Lindblad master equation $\dot{\rho}=-\frac{1}{2} \sum_{m=0}^{2}\left(\hat{L}_{m}^{\dagger} \hat{L}_{m} \rho+\right.$ $\left.\rho \hat{L}_{m}^{\dagger} \hat{L}_{m}-2 \hat{L}_{m} \rho \hat{L}_{m}^{\dagger}\right), \quad$ where $\quad \hat{L}_{0} \equiv \sqrt{\Lambda_{h}} \hat{J}_{T}^{-}$and $\hat{L}_{m} \equiv$ $\sqrt{\Lambda_{i}} \hat{J}_{m}^{+}$for $m=1,2$. Here, we have chosen the inhomogeneous raising operators,

$$
\begin{aligned}
& \hat{J}_{1}^{+}=e^{(2 / 3) \pi i} \hat{j}_{A}^{+}+e^{(4 / 3) \pi i} \hat{j}_{B}^{+}+\hat{j}_{\beta}^{+}, \\
& \hat{J}_{2}^{+}=e^{(4 / 3) \pi i} \hat{j}_{A}^{+}+e^{(8 / 3) \pi i} \hat{j}_{B}^{+}+\hat{j}_{\beta}^{+},
\end{aligned}
$$

while other choices of coefficients $c_{A, B, \beta}$ lead to similar results. For the simulated example, we set the spin pump rates $\Lambda_{h} / \Lambda_{i}=5000$ and $j_{A}=j_{B}=j_{\beta}=5$. After a pump time $t_{p}=0.2 \Lambda_{i}^{-1},\left|S_{A B \beta}\right\rangle$ is occupied by a population $P(0) \sim$ $20 \%$. For general values of $j_{A}, j_{B}$, and $j_{\beta}$, we require

$$
\Lambda_{h}\left\langle\hat{J}_{T}^{+} \hat{J}_{T}^{-}\right\rangle \gg \Lambda_{i}\left\langle\hat{J}_{i}^{-} \hat{J}_{i}^{+}\right\rangle
$$

which ensures the lowering operator $\hat{J}_{T}^{-}$to be applied much more frequently than the raising operator $\hat{J}_{i}^{+}$. The largest possible value of $\left\langle\hat{J}_{i}^{-} \hat{J}_{i}^{+}\right\rangle$is $\sim\left(j_{A}+j_{B}+j_{\beta}\right)^{2}$, while the smallest possible value of $\left\langle\hat{J}_{T}^{+} \hat{J}_{T}^{-}\right\rangle$is $\sim 1$. Thus, $\Lambda_{h} / \Lambda_{i} \gg$
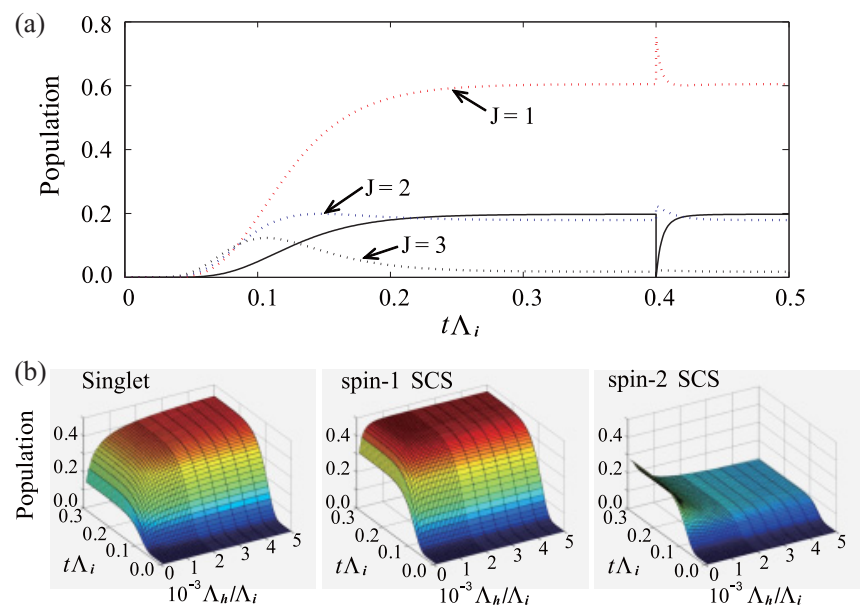

FIG. 1. (Color online) (a) Simulation of spin pump and repump by the collective operators $\hat{J}_{T}^{-}, \hat{J}_{1}^{+}$, and $\hat{J}_{2}^{+}$(see text). The target and ancilla spins are in the fully polarized state at $t=0$. (Solid curve) Population in the singlet $\left|S_{A B \beta}\right\rangle$. (Dotted curves) Populations in the subspace of $J=1$ (red), $J=2$ (blue), and $J=3$ (black), respectively. We assume the singlet is projected out at $t=0.4 \Lambda_{i}^{-1}$, and hence, the curve after is the repump dynamics. (b) Simulation of the simplified scheme for engineering the telecloning resource for $N=40$ qubits. The populations in the singlet $\left|0,0, \frac{N}{4}, \frac{N}{4}\right\rangle$, the spin- 1 $\operatorname{SCS}\left|1,-1, \frac{N}{4}, \frac{N}{4}\right\rangle$, and the spin-2 SCS $\left|2,-2, \frac{N}{4}, \frac{N}{4}\right\rangle$ are shown as functions of time using various pumping rates.

$\left(j_{A}+j_{B}+j_{\beta}\right)^{2}$ is sufficient to ensure the condition in Eq. (4). The steady-state population in $\left|S_{A B \beta}\right\rangle$ is given by $\left[\sum_{k} g(k)\right]^{-1}=20 \%$ where $g(k) \equiv(2 k+1) \prod_{i=0}^{k-1}\left(2 i^{2}+3 i+\right.$ $1)^{-1}$ [10]. The time scale to reach the steady state is $\sim\left(j_{A}+\right.$ $\left.j_{B}+j_{\beta}\right)^{-1} \Lambda_{i}^{-1}$. The singlet can be selected out by projective measurement of $\hat{J}_{T}^{z}$ or $\hat{J}_{T}^{2}$. If the measurement outcome is not the singlet, the spins can be repumped to the steady state in a much shorter time scale $\sim\left(j_{A}+j_{B}+j_{\beta}\right)^{-2} \Lambda_{i}^{-1}$ [Fig. 1(a)]. The probability of not obtaining $\left|S_{A B \beta}\right\rangle$ is reduced to $0.1 \%$ after 30 cycles of measure plus repump.

From the singlet $\left|S_{A B \beta}\right\rangle$, further pumping by the target spin operator $\hat{j}_{A}^{-}+\hat{j}_{B}^{-}$brings the target spins to the desired SCS $\left|J, \mu=-J, j_{A}=N_{A} I, j_{B}=N_{B} I\right\rangle$. Here, the collective spin value $J$ of the target spins is controlled by the number of the ancilla spins involved. Different choices of $N_{A}$ and $N_{B}$ realize distinct pure SCSs of the same collective spin value, which are fully symmetric under the permutation of spins within subgroup $A$ (or $B$ ). $A$ and $B$ can also be initialized with any $j_{A}<N_{A} I$ and $j_{B}<N_{B} I$ by applying the scheme first to the subgroups. Therefore, a concatenation of the scheme can realize pure SCSs with more general permutation symmetries.

SCSs of the smallest collective spin values are most desirable as a resource of entanglement. We consider the $J=0$ scenario of the above scheme (i.e., no ancilla spins), which uses two pump operators: the homogeneous $\hat{j}_{A}^{-}+\hat{j}_{B}^{-}$ and the inhomogeneous $\hat{j}_{A}^{+}-\hat{j}_{B}^{+}$, where $A$ and $B$ each contain $\frac{N}{2}$ target spins. For $\Lambda_{h} / \Lambda_{i} \gg N^{2}$, we find the steady state of the pumping $\rho=\sum_{J} P(J)\left|J,-J, \frac{N}{2} I, \frac{N}{2} I\right\rangle\left\langle J,-J, \frac{N}{2} I, \frac{N}{2} I\right|$ where $P(J)=\left(2 J^{2}+3 J+1\right) P(J+1)$. This steady state is reached with a pump time $t_{p} \approx \frac{3+\ln N I}{2 N I} \Lambda_{i}^{-1}$ by our numerical estimation and is largely a mixture of the singlet $\left|0,0, \frac{N}{2} I, \frac{N}{2} I\right\rangle$, 

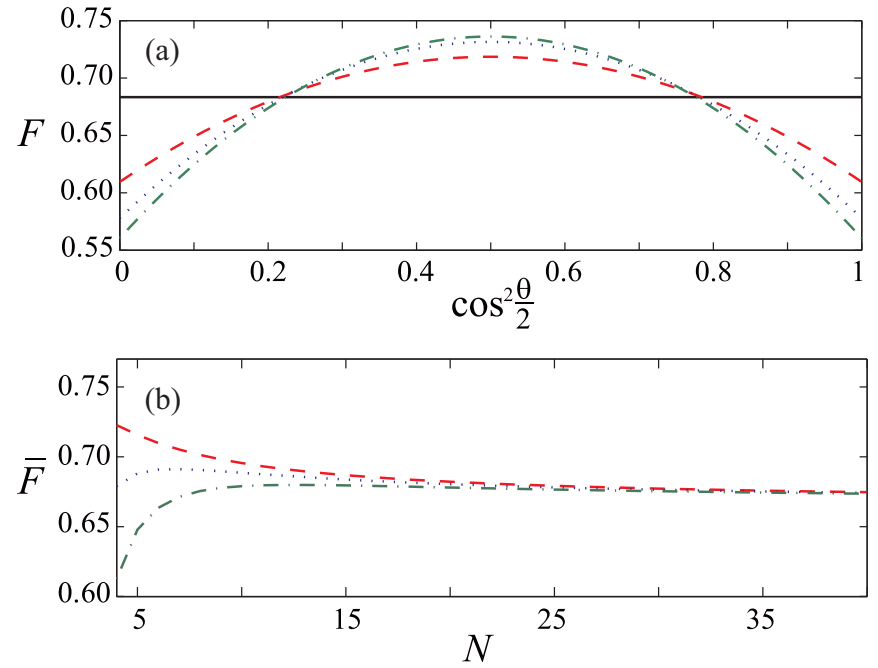

FIG. 2. (Color online) (a) Fidelity of $1 \rightarrow 20$ telecloning of a state $\cos \frac{\theta}{2}|0\rangle+\sin \frac{\theta}{2} e^{i \phi}|1\rangle$ using the above singlet (solid), spin-1 SCS (dashed), spin-2 SCS (dotted), and spin-3 SCS $\left|3,-3, \frac{N}{4}, \frac{N}{4}\right\rangle$ (dot-dashed) as the resource, respectively. (b) The fidelity of $1 \rightarrow \frac{N}{2}$ telecloning (averaged over the Bloch sphere) using the above resource states, respectively.

the spin-1 SCS $\left|1,-1, \frac{N}{2} I, \frac{N}{2} I\right\rangle$, and the spin-2 SCS $\mid 2$, $\left.-2, \frac{N}{2} I, \frac{N}{2} I\right\rangle$, with some residue population of $0.5 \%$ on the spin-3 SCS $\left|3,-3, \frac{N}{2} I, \frac{N}{2} I\right\rangle$. These states can be distinguished in a nondemolition way by measuring $\hat{j}_{A}^{z}+\hat{j}_{B}^{z}$. A single cycle of pump plus measure, thus, ends up with one of these pure states, which all have large-scale entanglement. Figure 1(b) shows a simulation of this spin pumping for a cluster of 40 spin qubits.

The singlet $\left|0,0, \frac{N}{4}, \frac{N}{4}\right\rangle$ of $N$ qubits turns out to be the ideal resource for universal optimal quantum telecloning [17]. If Alice holds subgroup $A$ and each of her $\frac{N}{2}$ associates holds a qubit in subgroup $B$, Alice can transmit identical copies of her unknown state $\cos \frac{\theta}{2}|0\rangle+\sin \frac{\theta}{2} e^{i \phi}|1\rangle$ with a fidelity of $F_{0}=\frac{2 N+2}{3 N}$ to the $\frac{N}{2}$ associates using local operations and classical communications (LOCCs) [17]. By a single cycle of pump plus measure, the success rate for obtaining this state is $\sim 46 \%$, which is a substantial improvement over the existing scheme where the success rate is $\frac{2}{2+N}$ [11]. Most remarkably, all alternative outcomes by our scheme, i.e., $\left|J,-J, \frac{N}{4}, \frac{N}{4}\right\rangle$ with a finite but small $J$, can also be used as quantum telecloning resources under the same LOCC. Following the same procedure of Ref. [17] but replacing $\left|0,0, \frac{N}{4}, \frac{N}{4}\right\rangle$ with $\left|J,-J, \frac{N}{4}, \frac{N}{4}\right\rangle$, we obtain the telecloning fidelity, which is then a function of $\theta$ [Fig. 2(a)], and it reaches the maximum value on the equator of the Bloch sphere,

$$
F_{J}^{\max }=\frac{(3 J+4) N^{2}+4(J+1) N-4 J(J+1)(J+2)}{2(2 J+3) N^{2}} .
$$

Since $F_{J}^{\max }>F_{0}$, better telecloning fidelity can be achieved with these finite $J$ SCSs in the presence of partial information (i.e., the range of $\theta$ value). For $N \gg J$, the telecloning
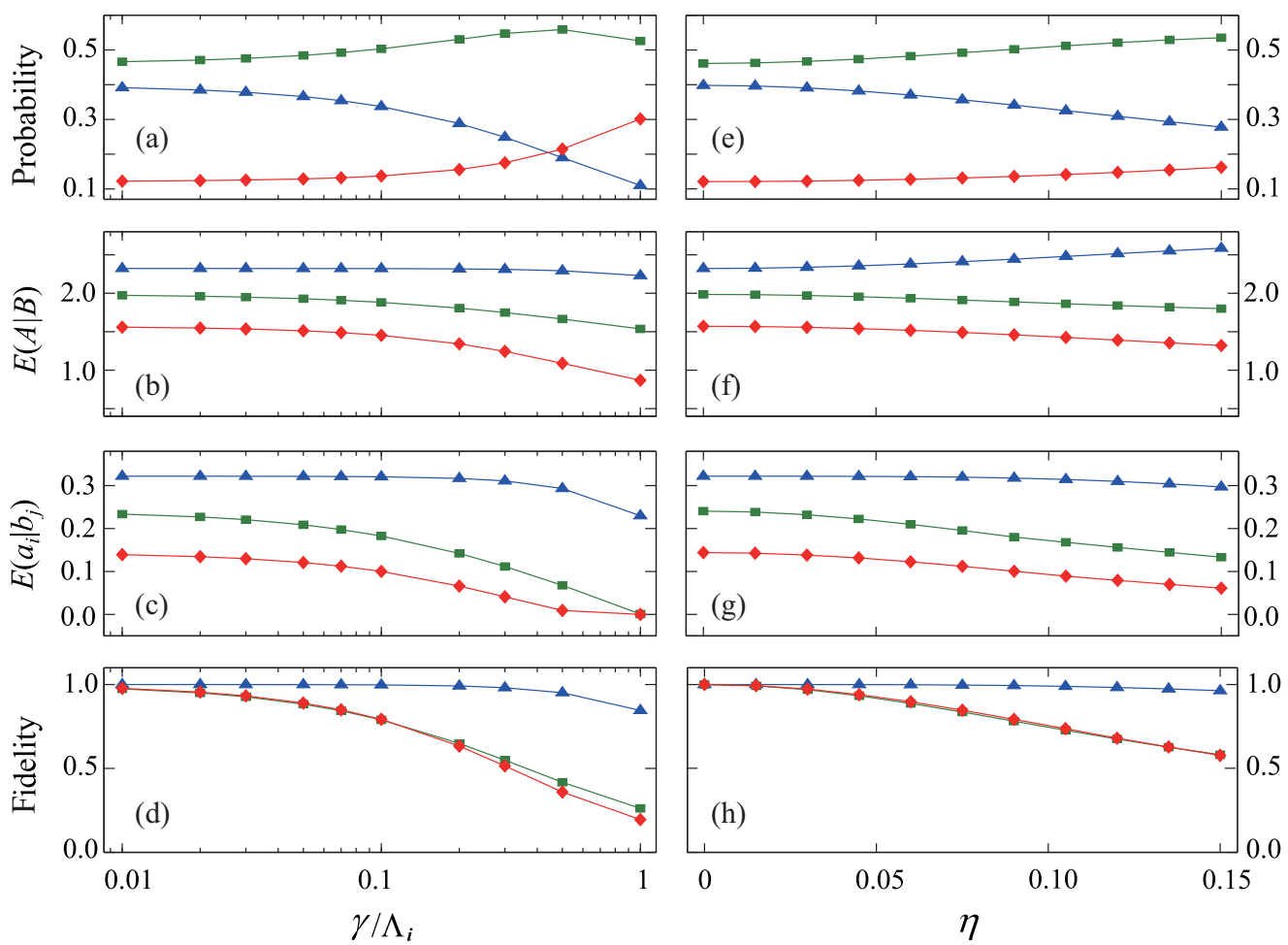

FIG. 3. (Color online) Merits of state engineering in the presence of spin decoherence (a)-(d) and system parameter errors (e)-(h) for engineering the telecloning resource for $N=8$ qubits. $\Lambda_{h} / \Lambda_{i}=100$. (a) and (e) Probability of obtaining singlet (blue triangles), spin-1 SCS (green squares), and spin-2 SCS (red diamonds) at $t_{p}=0.75 \Lambda_{i}^{-1}$. (b) and (f) Bipartite entanglement $E(A \mid B)$ in the obtained states measured with the logarithmic negativity. (c) and (g) Entanglement between one qubit in $A$ and another in $B$. (d) and (h) Fidelity of the obtained states with the target states $\left|0,0, \frac{N}{4}, \frac{N}{4}\right\rangle,\left|1,-1, \frac{N}{4}, \frac{N}{4}\right\rangle$, and $\left|2,-2, \frac{N}{4}, \frac{N}{4}\right\rangle$. 


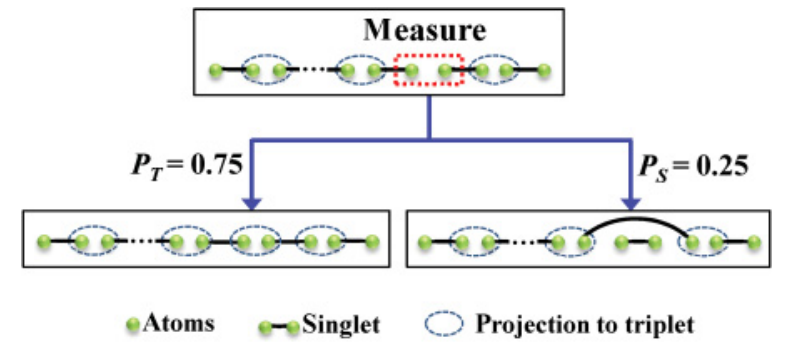

FIG. 4. (Color online) Increasing the length of an AKLT chain with the resource of a four-qubit-AKLT state. By measuring the parity of the two atoms, the $n$-qubit AKLT chain either becomes an $(n+4)$ qubit (lower left) or an $(n+2)$-qubit AKLT chain (lower right). See text.

fidelity averaged over the entire Bloch sphere approaches $F_{0}$ [Fig. 2(b)]. Thus, the mixed steady state of the spin pumping can be used as an equally efficient telecloning resource as the ideal singlet.

A major cause of error for the state engineering is the local spin decoherence process. If each spin loses its phase coherence with a rate $\gamma$, a total leakage of $\sim N \gamma t_{p} \sim \gamma / \Lambda_{i}$ out of the desired subspace is accumulated in the entire duration $t_{p} \sim \frac{1}{N} \Lambda_{i}^{-1}$ of the state preparation. High-fidelity state engineering, thus, requires $\gamma \ll \Lambda_{i}$. This is confirmed by numerical simulation for a cluster of $N=8$ spin qubits where we have added pure dephasing processes described by Lindblad operators $\sqrt{2 \gamma} \hat{I}_{n}^{z}$ for all spins [Figs. 3(a)-3(d)]. We also studied the effect of errors from system parameters. For the simulation presented in Figs. 3(e)-3(h), spins are pumped instead by $\hat{\Xi}_{A}^{-}+\hat{\Xi}_{B}^{-}$and $\hat{\Xi}_{A}^{+}-\hat{\Xi}_{B}^{+}$where $\hat{\Xi}^{ \pm} \equiv \sum_{n}(1+$ $\left.\eta_{n}\right) \hat{I}_{n}^{ \pm}, \eta_{n}$ being a random error between $\eta$ and $-\eta$. The figure of merit is reasonably good when the error amplitude $\eta<10 \%$. Moreover, by pumping with the operators $U\left(\hat{j}_{A}^{-}+\hat{j}_{B}^{-}\right) U^{\dagger}$ and $U\left(\hat{j}_{A}^{+}-\hat{j}_{B}^{+}\right) U^{\dagger}$ where $U \equiv \prod_{n} \exp \left(i \theta_{n} \hat{I}_{n}^{z}\right)$, state $U \mid J,-$ $\left.J, \frac{N}{2} I, \frac{N}{2} I\right\rangle$ is obtained instead of $\left|J,-J, \frac{N}{2} I, \frac{N}{2} I\right\rangle$. Namely, systematic phase errors in the collective pumping operators do not affect the entanglement, and single spin rotations about the $z$ axis can deliberately be encoded in the pumping.

When $A$ and $B$ each contain two qubits, the resultant singlet $|0,0,1,1\rangle$ by our scheme is the four-qubit AKLT state $P_{23}|S\rangle_{12}|S\rangle_{34}$, where $|S\rangle_{i j}$ stands for the singlet of qubits $i$ and $j$ and $P_{j k}$ is the projection operator to the triplet subspace for qubits $j$ and $k$ [25]. Its optical analog has been used to demonstrate four-party secret sharing [24] and measurement-based single-qubit rotation [26]. This state is also an efficient element for constructing a large-scale AKLT state as schematically illustrated in Fig. 4. Consider two four-qubit clusters in $P_{23}|S\rangle_{12}|S\rangle_{34}$ and $P_{67}|S\rangle_{56}|S\rangle_{78}$, respectively, by measuring the parity of atom pair $\{4,5\}$, the spin configuration of this pair will be projected to either the singlet or the triplet subspace [28]. With $75 \%$ probability, the measurement outcome is triplet and an eight-qubit AKLT chain $P_{23} P_{45} P_{67}|S\rangle_{12}|S\rangle_{34}|S\rangle_{56}|S\rangle_{78}$ is obtained. The remaining $25 \%$ probability will give $P_{23} P_{67}|S\rangle_{12}|S\rangle_{36}|S\rangle_{78} \otimes|S\rangle_{45}$ where a six-qubit AKLT state is obtained. With our scheme as an efficient source of four-qubit AKLT states, a long AKLT chain, thus, can be constructed.
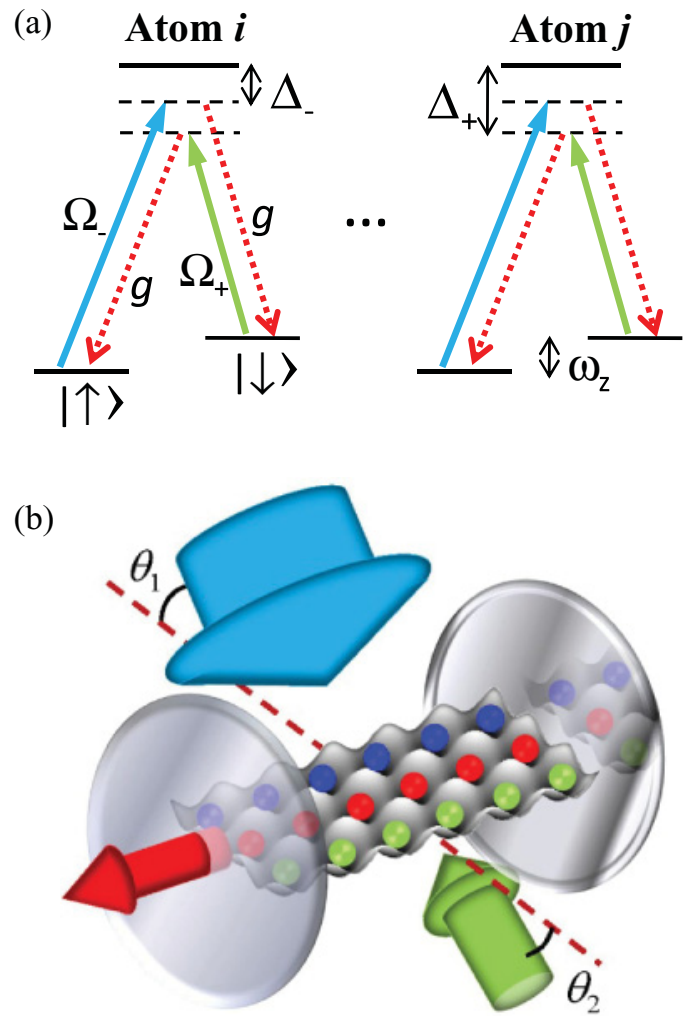

FIG. 5. (Color online) (a) The level structure of the atoms. The red dashed arrow represents the cavity field, and two solid arrows denote two laser fields. (b) The collective spin pumping of atoms realized by the cavity-assisted Raman process. Atoms are trapped in the optical lattice and are loaded into a Fabry-Pérot cavity. The inhomogeneous coefficients are controlled by laser emission angles $\theta_{1}$ and $\theta_{2}$.

\section{APPLICATION TO ATOMS IN AN OPTICAL LATTICE}

Here, we apply our scheme to cold atoms of a typical $\Lambda$-level structure, which are trapped in an optical lattice. This system has been explored widely in various schemes for quantum information processing. The two lower-energy levels are used to represent the spin qubit [Fig. 5(a)]. The atoms trapped in an optical lattice can first be initialized to the $|\downarrow\rangle$ state and can be loaded into a Fabry-Pérot cavity [Fig. 5(b)]. For simplicity, we assume the optical lattice constants in both directions equal to the wavelength of the cavity mode, and all atoms are at the peak of the cavity field. Driven by two lasers of frequency $\omega_{c} \pm \omega_{z}$ with $\omega_{c}$ being the cavity resonance and $\omega_{z}$ being the spin splitting, the cavity-assisted Raman process lowers or raises the spin state. The Raman processes in the large detuning regime $\left(g^{2}, \Omega_{ \pm}^{2} \ll \Delta_{ \pm}^{2}\right)$ can be described by the effective Hamiltonian,

$$
\hat{H}_{ \pm}=\frac{g \Omega_{ \pm}}{2 \Delta_{ \pm}} \hat{J}^{ \pm}(\mathbf{k}) \hat{a}_{c}^{\dagger}+\text { H.c. }
$$

where $\Omega_{ \pm}$is the Rabi frequency of the two pumping lasers, $g$ is the atom-cavity coupling, and $\Delta_{ \pm}$is the detuning. $\hat{a}_{c}^{\dagger}$ creates a cavity photon, and $\hat{J}^{ \pm}(\mathbf{k}) \equiv \sum_{j} e^{-i \mathbf{k} \cdot \mathbf{r}_{j}} \hat{\sigma}_{j}^{ \pm}$realizes various collective spin-raising and lowering-operations by controlling the laser wave vector k. For the setup shown in Fig. 5(b) where the pump lasers are perpendicular to the cavity axis, 
the blue laser with $\theta_{1}=\frac{\pi}{2}$ realizes the homogeneous operator $\hat{J}_{T}^{-}$, and the green laser with $\cos \theta_{2}=\frac{1}{3}$ (or $\frac{2}{3}$ ) realizes the inhomogeneous collective operator $\hat{J}_{1}^{+}$(or $\hat{J}_{2}^{+}$) where the subgroups $A, B$, and the ancilla are represented by spheres with different colors.

A projective measurement for selecting out the singlet state can be realized in the same setup. Applying a blue and a green laser with both $\theta=\frac{\pi}{2}$ and comparable Rabi frequency $\Omega_{-} \sim$ $\Omega_{+}$realizes the homogeneous raising and lowering operators $\hat{J}_{T}^{ \pm}$on the spin qubits. If the system is in a finite $J$ state, then Raman scatterings are allowed, and we will observe continuous cavity photon emission when $\hat{J}_{T}^{+}$and $\hat{J}_{T}^{-}$pump the spins. When the system is in a singlet, Raman scattering is forbidden since both $\hat{J}_{T}^{+}$and $\hat{J}_{T}^{-}$annihilate the state, and there will be no cavity photon emission.

The Raman-scattering rate by a single atom is $\Lambda_{h / i}=$ $P \Gamma \Omega_{ \pm}^{2} / \Delta_{ \pm}^{2}$ with $\Gamma$ being the spontaneous emission rate of the atomic excited state in vacuum and $P$ as the cavity-induced enhancement factor (Purcell factor). Consider the Cs atom $\left(\frac{\Gamma}{2 \pi}=2.6 \mathrm{MHz}\right)$ in a typical Fabry-Pérot cavity with a mode volume of $10^{4} \mu \mathrm{m}^{3}$ and a quality factor of $1.7 \times 10^{7}$, which correspond to $P \approx 80, \frac{g}{2 \pi} \approx 45 \mathrm{MHz}$, and a cavity-decay rate of $\frac{\kappa}{2 \pi} \approx 20 \mathrm{MHz}$ [29]. $\Lambda \approx 15 \mathrm{MHz}$ can then be achieved with $\frac{\Delta}{2 \pi} \approx 150$ and $\frac{\Omega}{2 \pi} \approx 40 \mathrm{MHz}$ [30]. The collective Ramanscattering rates should satisfy $\Lambda_{i}\left\langle\hat{J}_{1}^{-} \hat{J}_{1}^{+}\right\rangle \ll \Lambda_{h}\left\langle\hat{J}_{T}^{+} \hat{J}_{T}^{-}\right\rangle<\kappa$ [10]; the last inequality is to ensure that the emission of the cavity photon is spontaneous. For $N$ atoms in the cavity, the matrix element $\left\langle\hat{J}_{T}^{+} \hat{J}_{T}^{-}\right\rangle\left(\left\langle\hat{J}_{1}^{-} \hat{J}_{1}^{+}\right\rangle\right)$is $\sim N(\sim N)$ in the neighborhood of the polarized initial state and $\sim 1\left(\sim \frac{N^{2}}{4}\right)$ in the neighborhood of the target singlet state. Thus, we can use $\Lambda_{h} \sim 15 \mathrm{MHz}$. Correspondingly, $\Lambda_{i}$ will be ramped down from an initial value of $\sim 1 / N \mathrm{MHz}$ to the steady-state value $\sim \frac{1}{N^{2}} \mathrm{MHz}$ along with the spin pumping. Since an atom in an optical lattice can be of an ultraslow spin decoherence rate $\frac{\gamma}{2 \pi} \sim 1-25 \mathrm{~Hz}$ [31], the condition $\gamma \ll \Lambda_{i}$ can be satisfied for $N \sim O(100)$ qubits.

\section{ACKNOWLEDGMENTS}

The authors thank X. D. Xu for helpful comments. The work was supported by the Research Grant Council of Hong Kong under Grant No. 706711P.
[1] W. M. Zhang, D. H. Feng, and R. Gilmore, Rev. Mod. Phys. 62, 867 (1990).

[2] J. R. Glauber, Phys. Rev. Lett. 10, 84 (1963).

[3] J. R. Glauber, Phys. Rev. 130, 2529 (1963).

[4] E. C. G. Sudarshan, Phys. Rev. Lett. 10, 277 (1963).

[5] J. R. Klauder, J. Math. Phys. 4, 1055 (1963).

[6] A. M. Perelomov, Commun. Math. Phys. 26, 222 (1972).

[7] R. Gilmore, Ann. Phys. 74, 391 (1972).

[8] F. T. Arecchi, E. Courtens, R. Gilmore, and H. Thomas, Phys. Rev. A 6, 2211 (1972).

[9] G. Tóth and M. W. Mitchell, New J. Phys. 12, 053007 (2010).

[10] W. Yao, Phys. Rev. B 83, 201308 (2011).

[11] F. Ciccarello, M. Paternostro, S. Bose, D. E. Browne, G. M. Palma, and M. Zarcone, Phys. Rev. A 82, 030302 (2010).

[12] J. Kempe, D. Bacon, D. A. Lidar, and K. B. Whaley, Phys. Rev. A 63, 042307 (2001).

[13] D. Gottesman and I. L. Chuang, Nature (London) 402, 390 (1999).

[14] R. Raussendorf and H. J. Briegel, Phys. Rev. Lett. 86, 5188 (2001).

[15] A. S. Sørensen and K. Mølmer, Phys. Rev. Lett. 86, 4431 (2001).

[16] O. Gühne and G. Tóth, Phys. Rep. 474, 1 (2009).

[17] M. Murao, D. Jonathan, M. B. Plenio, and V. Vedral, Phys. Rev. A 59, 156 (1999).

[18] S. Diehl, A. Micheli, A. Kantian, B. Kraus, H. P. Buchler, and P. Zoller, Nat. Phys. 4, 878 (2008).
[19] F. Verstraete, M. M. Wolf, and J. I. Cirac, Nat. Phys. 5, 633 (2009).

[20] S. Clark, A. Peng, M. Gu, and S. Parkins, Phys. Rev. Lett. 91, 177901 (2003)

[21] S. Schneider and G. J. Milburn, Phys. Rev. A 65, 042107 (2002).

[22] J. Cho, S. Bose, and M. S. Kim, Phys. Rev. Lett. 106, 020504 (2011).

[23] M. J. Kastoryano, F. Reiter, and A. S. Sørensen, Phys. Rev. Lett. 106, 090502 (2011).

[24] S. Gaertner, C. Kurtsiefer, M. Bourennane, and H. Weinfurter, Phys. Rev. Lett. 98, 020503 (2007).

[25] I. Affleck, T. Kennedy, E. H. Lieb, and H. Tasaki, Phys. Rev. Lett. 59, 799 (1987).

[26] R. Kaltenbaek, J. Lavoie, B. Zeng, S. D. Bartlett, and K. J. Resch, Nat. Phys. 6, 850 (2010).

[27] A. S. Darmawan and S. D. Bartlett, Phys. Rev. A 82, 012328 (2010).

[28] A. M. Rey, V. Gritsev, I. Bloch, E. Demler, and M. D. Lukin, Phys. Rev. Lett. 99, 140601 (2007).

[29] C. J. Hood, T. W. Lynn, A. C. Doherty, A. S. Parkins, and H. J. Kimble, Science 287, 1447 (2000).

[30] M. Mitsunaga, T. Mukai, K. Watanabe, and T. Mukai, J. Opt. Soc. Am. B 13, 2696 (1996).

[31] U. Schnorrberger, J. D. Thompson, S. Trotzky, R. Pugatch, N. Davidson, S. Kuhr, and I. Bloch, Phys. Rev. Lett. 103, 033003 (2009); R. Zhao, Y. O. Dudin, S. D. Jenkins, C. J. Campbell, D. N. Matsukevich, T. A. B. Kennedy, and A. Kuzmich, Nat. Phys. 5, 100 (2008). 